\title{
Consideration of a buyback contract model that features game-leading marketing strategies
}

\author{
He, H. $^{\text {a, }}{ }^{,}{ }^{*}$, Jian, $M{ }^{a}{ }^{a}$, Fang, $X .{ }^{c}$ \\ ${ }^{a}$ School of Transportation and Logistics, Southwest Jiaotong University, Chengdu, China \\ ${ }^{\mathrm{b}}$ Tian Fu College of Southwestern University of Finance and Economics, Chengdu, China \\ 'School of Business Planning, Chongqing Technology and Business University, Chongqing, China
}

\section{A B S T R A C T}

Enterprises will sacrifice profits for market shares. For this reason, the maketo-stock upstream expects the downstream to order more. The paper argues the game leader sales-oriented upstream, motivating downstream make no shortage, and attempts to execute a buyback contract to reach realistic decisions. In this article, we research a supplier that is a sales-oriented leader and a retailer that is a profit-oriented follower. The retailer is required to order more than its optimal quantity. The primary analysis emphasizes either enhancing the buyback price or reducing the wholesale price. In the results, the buyback contract parameters are limited by both the sales-oriented supplier's retained earnings and the distribution of market demand. Numerical examples are given to illustrate contract parameters that affect the supply chain coordination, the order quantity of the retailer and the profit of the supply chain. The previous buyback contract literature assumes not only that the supplier and retailer are profit oriented but also that they achieve both supply-chain coordination and Pareto optimality. However, the paper discusses the parameters of the buyback contract when the supplier is sales oriented.
\end{abstract}

\section{ARTICLE INFO}

Keywords:

Buyback contract

Marketing strategy

Supply chain coordination

*Corresponding author:

9073637@qq.com

(He, H.)

Article history:

Received 12 June 2016

Revised 8 July 2016

Accepted 18 July 2016

\section{References}

[1] Chen, Y.G., Zhang, W.Y., Yang, S.Q., Wang, Z.J., Chen, S.F. (2014). Referral service and customer incentive in online retail supply chain, Journal of Applied Research and Technology, Vol. 12, No. 2, 261-269, doi: 10.1016/S16656423(14)72342-9.

[2] Pasternack, B.A. (1985). Optimal pricing and return policies for perishable commodities, Marketing Science, Vol. 4, No. 2, 166-176, doi: 10.1287/mksc.4.2.166.

[3] Cachon, G.P. (2003). Supply chain coordination with contracts, Handbooks in operations research and management science, Vol. 11, 227-339, doi: 10.1016/S0927-0507(03)11006-7.

[4] He, H., Fang, X., Du, Y. (2014). Revenue sharing contract design with marketing strategy types of suppliers. In: LISS 2014 - Proceedings of $4^{\text {th }}$ International Conference on Logistics, Informatics and Service Science, SpringerVerlag, Berlin Heidelberg, Germany, 275-280, doi: 10.1007/978-3-662-43871-8 42.

[5] Lee, H.L., Padmanabhan, V., Taylor, T.A., Whang, S. (2000). Price protection in the personal computer industry, Management Science, Vol. 46, No. 4, 467-482, doi: 10.1287/mnsc.46.4.467.12058.

[6] Yan, N.-N., Huang X.-Y. (2005). Returns policy model for supply chain with E-marketplace, Systems Engineering Theory Methodology Application, Vol. 14, No. 6, 492-496.

[7] Ding, D., Chen, J. (2008). Coordinating a three level supply chain with flexible return policies, Omega, Vol. 36, No. 5, 865-876, doi: 10.1016/i.omega.2006.04.004.

[8] Cai, J.H., Huang, W.L., Zhang, Z.G. (2008). Study on a two-echelon supply chain inventory model under buy-back contract, Journal of Industrial Engineering and Engineering Management, Vol. 22, No. 1, 122-124.

[9] Loch, C.H., Wu, Y. (2008). Social preferences and supply chain performance: An experimental study, Management Science, Vol. 54, No. 11, 1835-1849, doi: 10.1287/mnsc.1080.0910. 
[10] Ho, T.H., Su, X., Wu, Y. (2014). Distributional and peer-induced fairness in supply chain contract design, Production and Operations Management, Vol. 23, No. 2, 161-175, doi: 10.2139/ssrn.2246818.

[11] Lin, R.., Hou R. (2014). Experimental tests of buyback contract coordination and analysis for its failure, Journal of Management Science, Vol. 27, No. 3, 75-82.

[12] Zhang, Y., Donohue, K., Cui, T.H. (2015). Contract preferences and performance for the loss-averse supplier: Buyback vs. revenue sharing, Management Science, Vol. 62, No. 6, 1734-1754, doi: 10.1287/mnsc.2015.2182.

[13] Sluis, S., De Giovanni, P. (2016). The selection of contracts in supply chains: An empirical analysis, Journal of Operations Management, Vol. 41, 1-11, doi: 10.1016/i.jom.2015.10.002.

[14] Chen, J.X., Yu, C.H., Jin, L. (2004). Mathematical Analysis, Vol. 2, 94-96, High Education Press, Beijing, China.

[15] Ding, H., Guo, B., Liu, Z. (2011). Information sharing and profit allotment based on supply chain cooperation, International Journal of Production Economics, Vol. 133, No. 1, 70-79, doi: 10.1016/j.ijpe.2010.06.015.

[16] Tirole, J. (1997). Industrial Knowledge Theory, Vol. 1, 138-179, China People's Publishing House, Beijing, China.

[17] Xu, Z., Zhu, D.-L., Zhu, W.-G. (2008). Supply chain coordination under buy-back contract with sales effort effects, Systems Engineering - Theory \& Practice, Vol. 133, No. 1, 1-11. 


\title{
Obravnava modela pogodbe za povratni nakup, ki vpliva na vodilne trženjske strategije
}

\author{
He, H. ${ }^{a, b}{ }^{*}$, Jian, M. ${ }^{a}$, Fang, X. $^{c}$ \\ ${ }^{a}$ School of Transportation and Logistics, Southwest Jiaotong University, Chengdu, China \\ ${ }^{\mathrm{b}}$ Tian Fu College of Southwestern University of Finance and Economics, Chengdu, China \\ 'School of Business Planning, Chongqing Technology and Business University, Chongqing, China
}

\section{POVZETEK}

Podjetja se pogosto odpovedo dobičku zaradi tržnega deleža. Zaradi tega proizvajanje na zalogo pomeni pričakovanje večjega števila naročil. Prispevek razpravlja o vodilnih prodajno orientiranih proizvajalcih, ki spodbujajo prodajalce končnih izdelkov, da nimajo primanjkljajev in si prizadevajo za čim bolj dosledno izvrševanje pogodbe za povratni nakup. $\mathrm{V}$ tem prispevku raziskujemo dobavitelja, ki je prodajno usmerjen in trgovca, ki je usmerjen k dobičku. Od trgovca se pričakuje da naroča več, kot pa je njegova optimalna količina. $V$ glavni analizi je poudarek na izbiri med povečanjem cene povratnega nakupa ali znižanjem cene na debelo. Pri rezultatih so parametri povratnega nakupa omejeni z zaslužki prodajno orientiranega dobavitelja in z distribucijo trženjskih potreb. $V$ članku je za ilustracijo podan številčni primer, kjer pogodbeni parametri vplivajo na koordinacijo oskrbovalne verige, količino naročenega blaga s strani trgovca in dobiček oskrbovalne verige. Dosedanjih viri, ki govorijo o pogodbenem povratnem nakupu, ne privzemajo le, da sta dobavitelj in trgovec naravnana $\mathrm{k}$ dobičku, temveč tudi da usklajujeta oskrbovalno verigo in dosežeta Paretovo optimalnost. V pričujoči raziskavi pa teče razprava o parametrih modela povratnega nakupa, ko je dobavitelj prodajno usmerjen.
\end{abstract}

\section{PODATKI O ČLANKU}

Ključne besede:

Pogodba povratnega nakupa

Trženjska strategija

Usklajevanje oskrbovalne verige

*Kontaktna oseba:

9073637@qq.com

(He, H.)

Zgodovina članka:

Prejet 12. junija 2016

Popravljen 8. julija 2016

Sprejet 18. julija 2016 